\title{
Current Attitudes and Practices Around Screening Mammography Among Women in the United States: Results of a National Survey
}

$\mathrm{J}$ Gen Intern Med 36(6): 1802-4 DOI: $10.1007 / \mathrm{s} 11606-020-05892-1$

(c) Society of General Internal Medicine (This is a U.S. government work and not under copyright protection in the U.S.; foreign copyright protection may apply) 2020

\section{INTRODUCTION}

The optimal frequency of screening mammography (SM) is controversial. ${ }^{1-3}$ Professional societies' clinical practice guidelines differ despite being based on similar research evidence. ${ }^{1}$ The USA Preventive Services Task Force (USPSTF) recommends biennial whereas the American Cancer Society recommends annual SM, creating uncertainty about which guidelines to follow. ${ }^{2}$

To make informed decisions, women should be aware of guidelines and the benefits and risks of pursuing annual vs biennial SM. ${ }^{2}$ In a 2012 national survey, ${ }^{4}$ less than half of women knew that the USPSTF guidelines changed in 2009, and $12 \%$ knew that the recommendation changed from annual to biennial screening. No recent study has examined how frequently women in the USA undergo SM or their knowledge regarding guidelines. It is also unclear whether informing women of alternate guidelines would change their screening behaviors. If women are unwilling to adopt a new screening approach despite being informed, changes to guidelines may not actually impact screening practices. We therefore analyzed national survey data to examine current knowledge and use of SM.

\section{METHODS}

This is a subgroup analysis of a cross-sectional study assessing USA breast density laws; methods have been previously described. ${ }^{5}$ Briefly, in 2018 , we invited women aged 40-59 years with no history of breast cancer and $\geq 1$ prior SM to take an online survey which included items assessing frequency of SM use, awareness of biennial SM recommendations, and willingness to change to biennial screening. Figure 1 details the survey questions and responses.

We used multivariable logistic regression to estimate the association between awareness of biennial SM recommendations and willingness to change to biennial

Received March 9, 2020

Accepted April 30, 2020

Published online June 15, 2020
SM, controlling for sociodemographic factors and history of increased breast density. We used poststratification weights to account for oversampling of women with dense breasts and to generate nationally representative estimates.

\section{RESULTS}

Of the 10,112 women invited to participate in the main study, ${ }^{5}$ 6898 (68\%) completed the screener; we included responses from 2289 women who completed the full survey and had $>1$ SM in this subgroup analysis (Fig. 1). Respondents were 51 years old on average, mostly white, and mostly college educated (Table 1). The majority reported undergoing SM annually $(65 \%)$ whereas $23 \%$ reported biennial SM use (Fig. 1).

Of the women who reported annual SM use, 51\% were aware that some guidelines also recommend biennial SM as an option. Approximately $43 \%$ were unwilling to switch to biennial SM, even if physician recommended. There was no association between awareness of biennial SM recommendations and willingness to change to biennial SM (adjusted OR 0.80 [95\% CI $0.59,1.09])$. The most common reason for unwillingness to adopt biennial SM was belief that annual SM is superior at detecting breast cancer ( $46 \%$ of respondents) (Fig. 1).

\section{DISCUSSION}

Our national survey showed that $65 \%$ of women age 40-59 years who undergo SM did so annually. About half of these women were unaware that biennial screening is an alternative strategy, demonstrating that awareness of biennial SM recommendations may not have improved since last assessed in 2012. ${ }^{4}$ We found no association between awareness of these guideline recommendations and willingness to change to biennial SM, suggesting that promulgation of alternate and acceptable screening strategies alone may not change screening practices.

Many of the women who were unwilling to adopt biennial SM stated a concern about increased risk associated with this choice. However, it is unclear if these responses represent informed choices. ${ }^{3}$ Prior literature 
suggests that both women and physicians may not accurately understand the benefits and harms of annual vs biennial SM, impairing informed decision-making. ${ }^{6}$ Therefore, guidelines alone may not impact screening practices unless the benefits and risks of annual vs biennial SM are presented clearly to both patients and physicians. ${ }^{3}$ Since professional societies' screening recommendations vary, a greater emphasis on harmonizing guidelines might facilitate informed decision-making.
Our study has several limitations, including potential recall and social desirability bias and limited ability to infer causality from correlations. Additionally, the self-reported behavior in our study may differ from actual SM use. Finally, unmeasured confounding factors may have affected preference for annual screening, such as history of abnormal mammography.

In conclusion, most women who undergo SM in the USA report doing so annually. Only half of women were aware of biennial SM recommendations; even those who were aware were no more likely to change to biennial mammography.

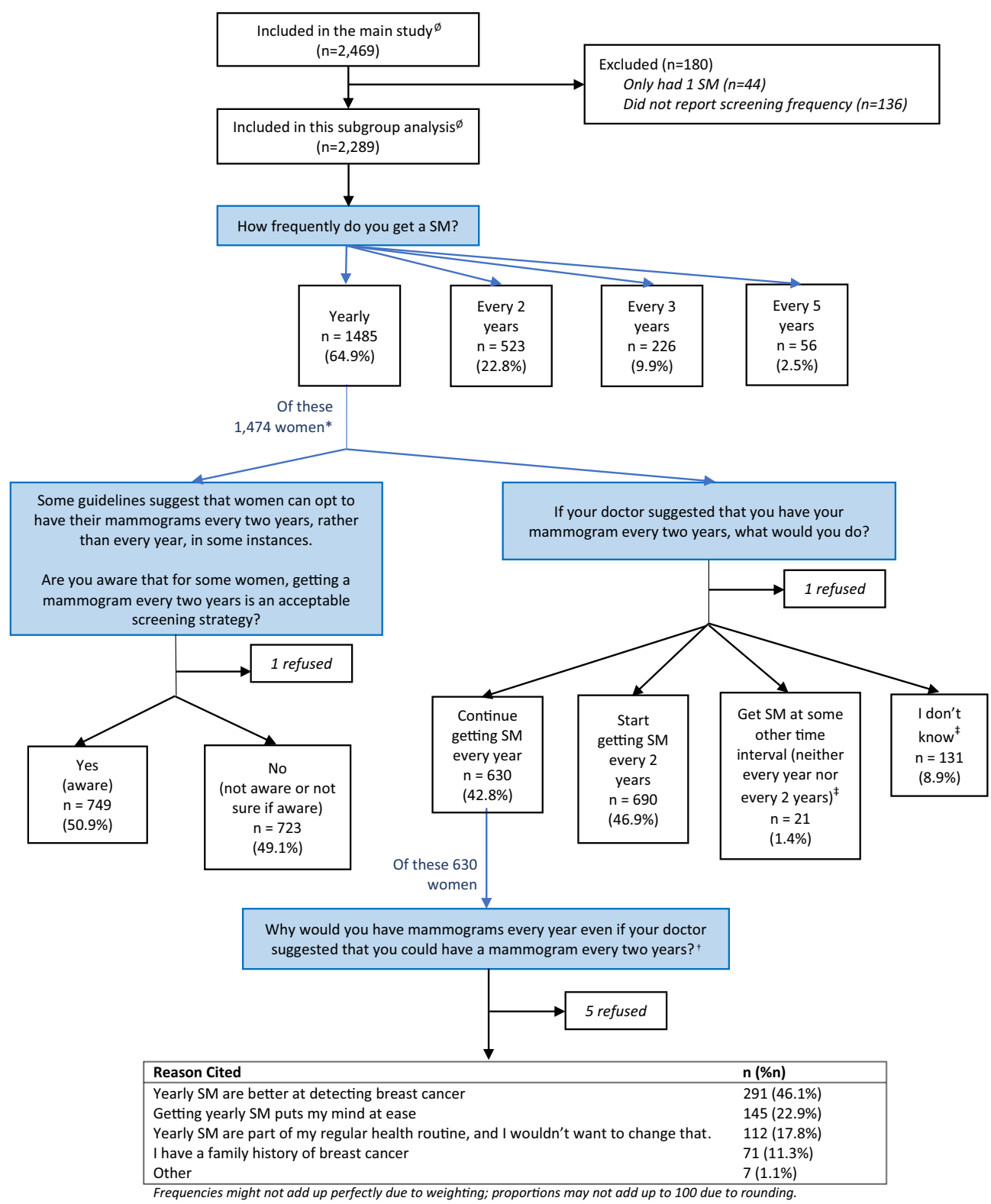

Fig. 1 Flow chart of survey questions, and responses. $\mathrm{SM}=$ routine, screening mammography/mammogram; $\mathrm{PCP}=$ primary care provider. All numbers are reported as weighted frequencies and may not add up perfectly due to rounding. Paragraph mark, The main study assessed knowledge of breast density reporting laws in the USA. The main study included women with only 1 prior SM or who reported undergoing SM, but did not report a clear screening interval. However, we excluded responses from these women in this subgroup analysis. Full details on the sample selection and reasons for excluding responses from the analysis are reported in the main study. ${ }^{5}$ Capital letter $\mathrm{O}$ with stroke, This is a subgroup analysis of a cross-sectional study assessing breast density laws in the USA. Asterisk, Due to the nature of survey skip logic, only those women who selected "yearly" $(n=1474)$ were invited to answer the subsequent questions about awareness and willingness to change SM use. The remaining women $(n=11)$ selected "other" but provided an answer consistent with annual SM use. Dagger, Respondents could select more than one answer to this question. Double dagger, these responses were not included in the multivariable logistic regression. 
Table 1 Characteristics of the Study Participants

\begin{tabular}{ll}
\hline \hline Characteristics & Percent \\
\hline Mean (standard deviation) age & 50.6 years (5.6 years) \\
Race and ethnicity & 65 \\
White & 12 \\
Black & 15 \\
Hispanic & 9 \\
Other & 8 \\
Education & 25 \\
< High school & 32 \\
High school & 21 \\
Some college or associate's degree & 14 \\
Bachelor's degree & \\
Graduate (master's or doctorate) degree & 16 \\
Insurance & 79 \\
Public & 5 \\
Private/other & \\
No insurance &
\end{tabular}

Frequencies are reported as weighted proportions and may not add up to 100 due to rounding

Mia Djulbegovic, $M D^{1,2}$

Jenerius Aminawung, $M D, M P H^{3}$

Jessica R. Hoag, PhD, MPH

Kelly A. Kyanko, MD, $M H S^{5}$

Xiao $\mathrm{Xu}, \mathrm{PhD}, M A^{3,6,7}$

Susan H. Busch, $P h D^{3,8}$

Cary P. Gross, $M D^{1,3}$

${ }^{1}$ National Clinician Scholars Program, School of Medicine, Yale University,

New Haven, CT, USA

${ }^{2}$ Veterans Affairs Connecticut Healthcare System,

West Haven, CT, USA

${ }^{3}$ Cancer Outcomes, Public Policy, and Effectiveness Research (COPPER) Center, Yale School of Medicine,

New Haven, CT, USA

${ }^{4}$ ICON plc.,

New Haven, CT, USA

${ }^{5}$ Department of Population Health, School of Medicine, New York University,

New York City, NY, USA

${ }^{6}$ Department of Obstetrics, Gynecology and Reproductive Sciences, School of Medicine, Yale University,

New Haven, CT, USA

${ }^{7}$ Yale Center for Outcomes Research \& Evaluation (CORE),

New Haven, CT, USA
${ }^{8}$ Department of Health Policy and Management, Yale School of Public Health, New Haven, CT, USA

Corresponding Author: Mia Djulbegovic, MD; Veterans Affairs Connecticut Healthcare System West Haven, CT, USA (e-mail: mia.djulbegovic@yale.edu).

Funding Information Financial support for this research was awarded to Dr. Gross (senior author) by the American Cancer Society (grant RSGI-15-151-01). Additional support was provided to Dr. Mia Djulbegovic (first author) by CTSA Grant Number TL1 TROO1864 from the National Center for Advancing Translational Science (NCATS), a component of the National Institutes of Health (NIH). These funds were provided to Dr. Djulbegovic by the National Clinician Scholars Program at Yale University School of Medicine. The content of this research is solely the responsibility of Dr. Djulbegovic and the author team and do not necessarily represent the official view of NIH. Dr. Djulbegovic is also sponsored by the Veterans Affairs Connecticut Healthcare System. We have no other disclosures to report.

\section{Compliance with Ethical Standards:}

Conflict of Interest: Dr. Gross has received research funding through Yale from Johnson \& Johnson and Pfizer. In addition, Dr. Gross has received compensation from Flatiron Health for travel and speaking.

\section{REFERENCES}

1. Shekelle PG. Clinical practice guidelines: What's next? JAMA. 2018.

2. Keating NL, Pace LE. Breast cancer screening in 2018: time for shared decision making. JAMA. 2018;319(17):1814-1815.

3. Gigerenzer G. Full disclosure about cancer screening. BMJ. 2016;352:h6967.

4. Kiviniemi MT, Hay JL. Awareness of the 2009 US Preventive Services Task Force recommended changes in mammography screening guidelines, accuracy of awareness, sources of knowledge about recommendations, and attitudes about updated screening guidelines in women ages 40-49 and 50+. BMC Public Health. 2012;12:899-899.

5. Kyanko KA, Hoag J, Busch SH, et al. Dense breast notification laws, education, and women's awareness and knowledge of breast density: a nationally representative survey. J Gen Int Med. 2020:1-6.

6. Wegwarth $\mathbf{O}$, Gigerenzer G. The barrier to informed choice in cancer screening: statistical illiteracy in physicians and patients. In: Goerling U, Mehnert A, eds. Psycho-Oncology. Cham: Springer International Publishing; 2018:207-221.

Publisher's Note: Springer Nature remains neutral with regard to jurisdictional claims in published maps and institutional affiliations. 